\title{
Neue Decklack-Linie für Lotus
}

\author{
Am britischen Stammsitz von Lotus wird im Zuge der Anlagenmodernisierung eine automatisierte \\ Decklacklinie einschließlich Sonderfarbversorgung und Fördertechnik gebaut. Den Auftrag dazu \\ erhielt Dürr im letzten Jahr.
}

Der traditionsreiche Sportwagenhersteller Lotus automatisiert seine Karosserielackierung am britischen Stammsitz Hethel. Bei der Vergabe des Auftrags konnte Dürr sich gegen die weltweite Konkurrenz durchsetzen und mit besonders effizienten und umweltschonenden Technologien überzeugen. Die Modernisierung umfasst eine automatisierte Decklacklinie einschließlich Sonderfarbversorgung und Fördertechnik. Bislang werden alle Fahrzeuge bei Lotus von Hand lackiert, doch zukünftig sollen vier Lackierroboter vom Typ EcoRP E133 die Außenlackierung übernehmen. Mit ihren sechs Achsen plus Verfahrschiene sind die Roboter für unterschiedliche Anwendungen flexibel einsetzbar. Bei der Konzeption der neuen Lackieranlage legte Lotus Wert auf eine besonders hohe Prozesseffizienz, ohne die hohe Qualität des bisherigen manuellen Lackierprozesses zu mindern.

Die mit einer leitfähigen Beschichtung grundierten Karosserieteile aus Verbundwerkstoff wird ein Roboterpaar mit Primer und Klarlack in einer Kombizone beschichten; ein zweites Roboterpaar wird in einer weiteren Kabine den farbgebenden Basislack auftragen. Durch ihre modulare Konstruktion können die Roboter nicht nur lackieren, sondern auch die Hauben der Karosserien öffnen.

\section{Sparsam und schnell: Sonderfarb- versorgung mit Molchsystem}

Die hohe Effizienz und Qualität wird nicht nur durch den Einsatz von Robotern erreicht, sondern auch durch Technologien mit geringem Ressourcenverbrauch. Jedes Lotus-Fahrzeug wird sorgfältig nach den individuellen Anforderungen fertiggestellt, einschließlich einer fast unendlichen Anzahl von Farben, die bei allen Modellreihen zur Auswahl stehen. Individuelle Besonder-

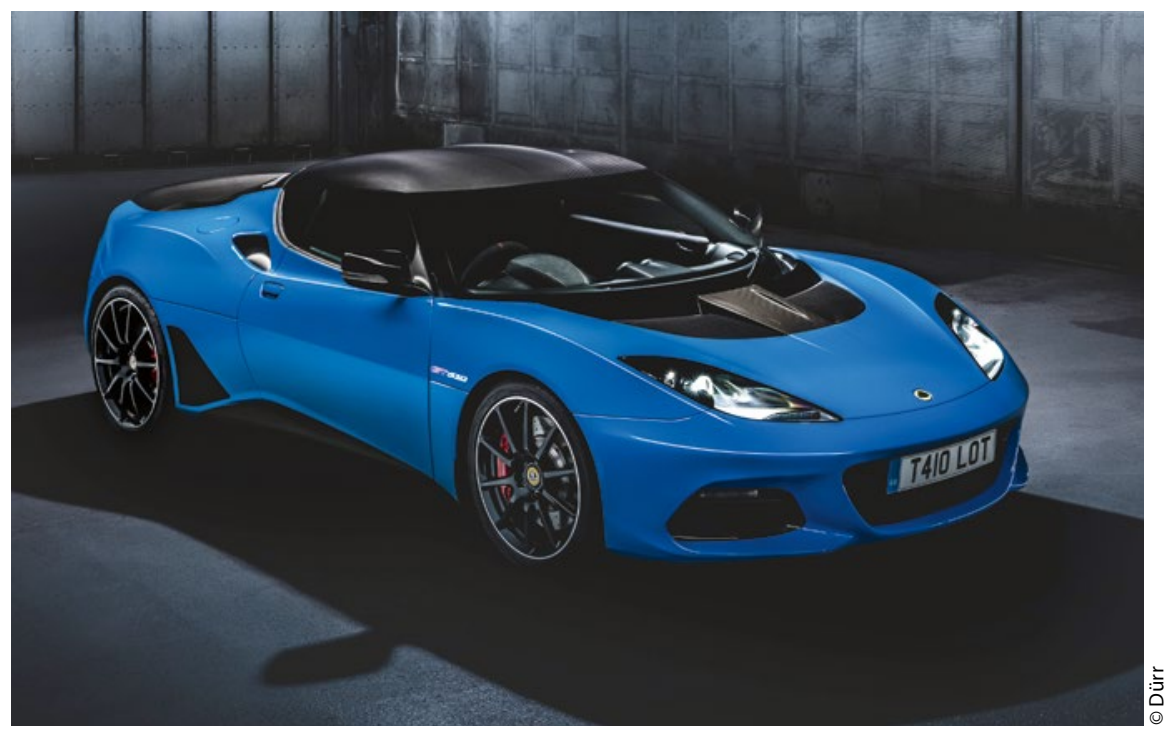

Die Sportwagen von Lotus, wie der Evora, werden zukünftig mit Lackierrobotern von Dürr lackiert.

heiten wie Streifen und Highlights werden weiterhin manuell lackiert.

Um diese große Farbvielfalt bei geringen Stückzahlen bereithalten und effizient applizieren zu können, kommt die Sonderfarbversorgung EcoSupply P mit drei Molchsystemen zum Einsatz. Diese befördern die diversen Farben in Schläuchen zur Lackierkabine - nahezu ohne Verlust, wodurch Lack eingespart wird. Außerdem minimiert das System den Spülmittelverbrauch und die Farbwechselzeiten im Vergleich zu herkömmlichen Ringleitungssystemen.

Zur Effizienzsteigerung trägt auch das Filtersystem EcoDry Rack für Overspray bei, das durch festinstallierte Filterwände besonders platzsparend ist und alle Vorteile der Trockenabscheidung besitzt. Es benötigt weder Wasser noch Chemie, was die laufenden Kosten gering hält und die Umwelt schont. Mit dieser Technologie können bis zu 95 Prozent der Prozessluft als Um- luft geführt werden und so den Energieverbrauch in der Lackierkabine im Vergleich zu einer klassischen Nassauswaschung um mehr als 60 Prozent reduzieren. Dies wirkt sich wiederum positiv auf die $\mathrm{CO}_{2}$-Emissionen aus.

Das mehrstufige Filtersystem ist einfach $\mathrm{zu}$ bedienen und $\mathrm{zu}$ warten. Sind die Filter gesättigt, lassen sie sich üblicherweise in den regulären Produktionspausen austauschen. Anschließend können sie thermisch entsorgt werden. Die neue Lackieranlage soll 2021 bei Lotus in Betrieb genommen werden. //

\section{Kontakt}

Dürr Systems AG, Bietigheim-Bissingen Kristin Roth, Marketing, kristin.roth@durr.com www.durr.com 